\title{
Patient characteristics of new initiators of glycopyrronium bromide (GLY) - A multinational, multi-database real-world drug-utilization study
}

Katia M.C. Verhamme ${ }^{1}$, Maria De Ridder ${ }^{1}$, Lars Pedersen ${ }^{2}$, Daniel Prieto-Alhambra ${ }^{3,6}$, Francesco Lapi $^{4}$, Peter Rijnbeek ${ }^{1}$, Rajeev K. Amar ${ }^{5}$ and Miriam Sturkenboom ${ }^{1}$

${ }^{1}$ Medical Informatics, ErasmusMC, Rotterdam, Netherlands

${ }^{2}$ Department of Clinical Epidemiology, Aarhus University, Aarhus, Denmark

${ }^{3}$ Idiap Jordi Gol Primary Care Research Institute, Universitat Autonoma de Barcelona, Barcelona, Spain

${ }^{4} \mathrm{HSD}$, Italian College of General Practitioners and Primary Care, Firenze, Italy

${ }^{5}$ Quantitative Safety \& Epidemiology - DS\&E, Novartis Pharma AG, Basel, Switzerland

${ }^{6}$ Musculoskeletal Pharmaco- and Device Epidemiology, University of Oxford, Oxford, United Kingdom

Background: GLY is a long-acting muscarinic antagonist (LAMA) indicated as maintenance bronchodilator to relieve symptoms in adults with COPD. Patient characteristics in real life often differ from clinical trials.

Aim: To describe demographics, dosing, duration of use, COPD severity, comorbidities and prior use of respiratory drugs in GLY initiators.

Methods: GLY initiators were identified in 5 electronic databases: UK (THIN), The Netherlands (IPCI), Denmark (Aarhus), Italy (HSD) and Spain (SIDIAP). The study period was from 2012-2015. Patients with at least $1 \mathrm{yr}$ of history were eligible. Using the pooled-study population, descriptive statistics were computed.

Results: $13,707 \mathrm{GLY}$ initiators were identified, $8 \%$ in IPCI, 10\% in Aarhus, $16 \%$ in THIN, $21 \%$ in HSD and $45 \%$ in SIDIAP. The median age was 71 yrs (IQR 62.9-78.7) and almost $60 \%$ were male. More than $98 \%$ used GLY once daily with a median duration of 61 days (IQR 30-183). COPD severity in patients with spirometry (41\%) was as follows: $9 \%$ mild, $49 \%$ moderate, $36 \%$ severe and $6 \%$ very severe. Cardio- and cerebrovascular comorbidity was high with highest prevalences for cardiac arrhythmia (14\%), heart failure (9\%), angina pectoris (7\%) and stroke (7\%). More than half of patients had chronic kidney disease stage 2 or 3.1 patient in 4 used LAMA (28\%) or LABA (26\%) in the 6 months prior to GLY start; prior use of LABA+ICS was even higher (46\%).

Conclusion: In real life, majority of first-time GLY use was in line with product label regarding dosing and COPD severity. More than $1 / 4$ of patients used long-acting bronchodilators before GLY start. Cardiovascular comorbidity was considerable. 